\title{
A formação docente inicial no PIBID-Artes Visuais/UFPel - apontamentos experienciais
}

\author{
Formación inicial en PIBID Artes Visuales / UFPel - apuntes \\ experienciales
}

Initial teacher formation in PIBID Visual Arts / UFPel - Experiential notes

\author{
Maristani Polidori Zamperetti ${ }^{1}$
}

\begin{abstract}
Resumo
Através desse artigo busco fazer um relato de uma pesquisa em andamento que reflete sobre a importância das atividades do Subprojeto Artes Visuais do Pibid/UFPel na formação de futuros professores de Artes Visuais, na Universidade Federal de Pelotas. A partir de textos - relatórios, avaliações e autoavaliações realizadas no primeiro semestre de 2015 e o desenvolvimento de subprojetos durante o ano - busco indícios qualitativos da inserção do Pibid nas escolas, colaborando na formação docente dos acadêmicos. Percebo, pelos dados apresentados, que o Projeto tem gerado boa receptividade do contexto escolar em relação aos acadêmicos do curso, ocasionando aprendizagens no que tange às suas inserções na realidade escolar. A prática investigativa tem sido estimulada no grupo e é possível observar uma aproximação maior dos bolsistas com a escrita acadêmica e a necessidade de registros de suas práticas. Desta forma, a pesquisa chega à sala de aula e o acadêmico promove a integração das diversas áreas do conhecimento em uma perspectiva interdisciplinar. Assim, a colaboração do Pibid na parceria Escola-Universidade se mostra na conjugação dos diferentes contextos, proporcionando reverberações nos dois campos de atuação para os universitários e seus professorescoordenadores, como também para os professores-supervisores e seus alunos.
\end{abstract}

Palavras-Chave: Artes Visuais; Formação Docente; Licenciaturas; PIBID; UFPel.

\section{Resumen}

A través de este artículo te daré una cuenta de una investigación en curso que reflexiona sobre la importancia de las actividades de los subproyectos de Artes Visuales PIBID / UFPel en la formación de los futuros profesores de Artes Visuales de la Universidad Federal de Pelotas. A partir de los textos - informes, evaluaciones y autoevaluaciones llevadas a cabo en la primera mitad de 2015 y el desarrollo de sub-proyectos durante el año - buscar evidencia cualitativa de inserción de PIBID en las escuelas, trabajando en la formación docente de los académicos. Me he dado cuenta, por los datos presentados, el proyecto ha generado buena receptividad del contexto escolar en relación con el curso académico, lo que lleva al aprendizaje en relación con sus inserciones en la realidad escolar. La práctica de la investigación ha sido estimulado en el grupo y es posible observar más de cerca los estudiosos con la escritura académica y la necesidad de registros de sus prácticas. Por lo tanto, la búsqueda llega al aula académica y promueve la integración de las diferentes áreas del conocimiento en una perspectiva interdisciplinaria. Por lo tanto, la colaboración de todos se muestra en la combinación de diferentes contextos, proporcionando reverberaciones en los dos campos de actividad para los estudiantes y sus profesores, ingenieros, así como para los profesores, supervisores y sus estudiantes.

Palabras claves: Artes Visuales; Formación del Profesorado; Licenciatura; PIBID; UFPel.

\section{Abstract}

Through this article I give an account of an ongoing research that reflects on the importance of the Sub-project activities Visual Arts Pibid / UFPel the training of future teachers of Visual Arts at the Federal University of Pelotas. From texts - reports, evaluations and self-assessments carried out in the first half of 2015 and the

\footnotetext{
${ }^{1}$ Doutora em Educação; Universidade Federal de Pelotas; Pelotas, Rio Grande do Sul, Brasil; maristaniz@hotmail.com.
} 
development of sub-projects during the year - seek qualitative evidence of insertion of Pibid in schools, working in teacher training of academics. I notice, by the data presented, the project has generated good receptivity of the school context in relation to the academic course, leading to learning in relation to their insertions in the school reality. The research practice has been stimulated in the group and it is possible to observe closer the scholars with academic writing and the need for records of their practices. Thus, the search comes to the classroom and academic promotes the integration of different areas of knowledge in an interdisciplinary perspective. Thus, the collaboration of Pibid School-University Partnership shown in combination of different contexts, providing reverberations in the two fields of activity for the students and their teachers, engineers, as well as for teachers, supervisors and their students.

Keywords: Visual Arts; Teacher Training; Undergraduate; PIBID; UFPel

\section{Introdução}

O presente artigo apresenta um recorte de pesquisa em andamento que está sendo realizada no Grupo de Pesquisa "Pesquisa, Ensino e Formação Docente nas Artes Visuais" (CNPQ), Centro de Artes, Universidade Federal de Pelotas (UFPel), RS. A mesma investiga a importância das atividades do Subprojeto Artes Visuais do Pibid/UFPel (Projeto Institucional de Bolsas de Iniciação à Docência) na formação de futuros professores de Artes Visuais.

Entende-se, hoje, que a formação de um professor é um processo contínuo e dinâmico, produzido, dentre outros fatores, pelos professores nas vivências e experiências de sala de aula (NÓVOA, 1997; 2009). Faz parte da formação humana, com tempos variados, de acordo com os períodos de vida e necessidades individuais e grupais dos sujeitos em formação. Confunde-se, por vezes, com a própria vida e as vivências humanas dos sujeitos, embora as múltiplas variações dos tempos cronológicos não coincidam com o transcurso dos dias letivos escolares, fazendo com que estejamos sempre no vir-a-ser, no vir-a-se-tornar alguém (ASSMANN, 2004).

O momento do ingresso do futuro licenciando na formação inicial é o primeiro passo de sua trajetória, pois como sustenta Freire (2004), somos "seres inacabados", em permanente construção e formação. Assim, a formação do professor é constante, pois a cada dia, novas situações acontecem e diferentes formas de vê-las são necessárias para o redirecionamento de sua ação docente.

Porém, sabemos que os cursos de licenciatura, em sua maioria, caracterizam-se pela dicotomia teoria-prática e pela falta de integração entre as disciplinas específicas da área de conhecimento e as disciplinas pedagógicas. Seguem uma tendência tradicional, conferindo, por vezes, uma formação fragmentada e simplista (PIMENTA, 2005). Ainda que as instituições formadoras busquem a superação dessa situação, a formação de professores ainda é um desafio para os docentes universitários que lidam diretamente com as situações críticas, diante das realidades educacionais. 
RELACult - Revista Latino-Americana de Estudos em Cultura e Sociedade

Revista Latinoamericana de Estudios en Cultura y Sociedad | Latin American Journal of Studies in Culture and Society

V. 02, no 03, set-dez., 2016, p. 22-32 | periodicos.claec.org e-ISSN Atual: 2525-7870 | e-ISSN Anterior: 2447-018X

$\mathrm{Na}$ área do Ensino das Artes Visuais, a formação do professor necessita ser reflexiva a partir de uma base sólida de conhecimentos específicos, pois a escola carece

[...] de um profissional que esteja aberto e qualificado para lidar com diferentes realidades e diversidade: cultural e educacional, situações de ensino, espaços e contextos, o que inclui o domínio de conhecimentos históricos, estéticos, artísticos, processuais, técnicos, de criação e processos de ensinar e aprender. Ele deve conhecer os materiais, os aspectos conceituais e filosóficos: da educação e da arte, mas sem se esquecer da complexidade e diversidade cultural que envolve todas as temáticas, práticas e contextos escolares (GOYA, 2015).

Desta forma, o Pibid como um programa de formação docente, é o contexto percebido, pelos bolsistas e por mim, coordenadora do subprojeto Artes Visuais, como uma possibilidade de inserção na prática profissional deste futuro docente, tornando-o um agente renovador e atuante no processo de transformação sociocultural, a fim de propiciar meios para uma ressignificação da escola.

A pesquisa parte de textos em forma de relatórios, avaliações e autoavaliações realizadas no primeiro semestre de 2015 e do desenvolvimento de subprojetos, onde buscamos indícios qualitativos da inserção do Pibid nas escolas, colaborando na formação docente dos acadêmicos.

\section{Desenvolvimento}

O segundo semestre de atuação dos pibidianos na escola se caracterizou pela implementação de subprojetos criados pelos próprios acadêmicos e orientados por mim, coordenadora. Estas pequenas inserções, algumas em forma de oficinas, tinham como finalidade estimulá-los a desenvolver e investigar temas de seus interesses e outros, trazidos para as reuniões de estudos do grupo. Algumas temáticas atravessavam os conteúdos específicos das diferentes disciplinas; tratavam-se dos Temas Transversais, presentes nos PCN (BRASIL, 1996), considerados como “questões urgentes que devem necessariamente ser tratadas, como a violência, a saúde, o uso de recursos naturais, os preconceitos, que não têm sido contempladas [pelas] áreas" (BRASIL, 1997, p. 23).

Apesar dos PCN assumirem que existam maiores afinidades de determinados temas com áreas específicas, o mesmo, ao justificar a necessidade da abordagem dos Temas Transversais, baseia-se

na ideia de que a organização disciplinar é uma das principais responsáveis pela pouca relevância social dos conhecimentos tratados pela escola. Defendem que a sociedade e os modos de produção estão cada dia mais complexos e globais e que a estruturação disciplinar do conhecimento corresponde a uma etapa inicial do modo 
de produção capitalista baseado na divisão linear do trabalho (MACEDO, 1998, p. 23).

Os subprojetos dos bolsistas, além de favorecerem um espaço de expressão e experimentação de metodologias e temáticas relevantes, apresentavam, por parte da coordenação, uma preocupação com a melhoria da qualidade de suas formações. Entendemos que, ao desenvolverem atividades diversificadas e incentivadoras, os bolsistas estariam se preparando para as realidades complexas com as quais se defrontariam, em contato com os projetos interdisciplinares e as escolas. Diversas atividades foram desenvolvidas em outros espaços educacionais, como museus e casas de abrigo para crianças, e em atividades destinadas à acadêmicos da universidade. Ainda que estes não fossem o foco principal das nossas atividades, estas ações foram pensadas pelos próprios acadêmicos, como uma forma de ampliação de espaços de aprendizagem e de atuação dos futuros docentes de Artes Visuais.

As relações com o Meio Ambiente, Trabalho e Consumo têm sido trabalhadas no grupo, conforme foi notificado em texto apresentado no II Seminário Internacional Ensino da Arte2, realizado no Centro de Artes/UFPel, em outubro de 2015. Na apresentação do trabalho realizado pela bolsista Amanda foi apontado, em relação às atividades desenvolvidas no ano, que

[o]s grupos passaram a realizar experimentações artísticas, apropriando-se espontaneamente de objetos descartados durante o cotidiano, para a execução de suas respectivas oficinas. Colaboradores do Pibid na oficina de gravura, voluntariamente se reapropriaram de diversos objetos que muitas vezes são descartados, utilizando bandejas de isopor e tampas de garrafas como carimbos/matrizes de impressão. [...] O uso de materiais orgânicos encontrados nas ruas da cidade, como cascas de árvores, foram empregadas na confecção de máscaras durante a semana da Consciência Negra nas escolas de ensino fundamental atendidas pelo Pibid/UFPel (SOUZA, ZAMPERETTI, 2015, p. 329).

Outro ponto que foi ressaltado nas avaliações foi a importância do início dos projetos interdisciplinares nas escolas, fazendo com que os bolsistas das Artes Visuais tivessem que estabelecer relações e conexões com outras áreas de conhecimento. No entender de Cibele, a percepção da importância da sua "[...] participação nos eventos oferecidos pela faculdade, [os quais] foram muito produtivos e me abriram um grande leque de referências para minha formação, foi um ponto positivo a ser destacado". Outros bolsistas relatam ainda que sentiram valorizados ao perceber a importância de seu trabalho na escola, destacando um certo

\footnotetext{
${ }^{2} \mathrm{O}$ evento pretendia consolidar e ampliar o espaço crítico de reflexão e o debate em torno dos temas relacionados à Arte e seu ensino, à Cultura Visual, às poéticas do cotidiano, à educação e à escola em pesquisa interdisciplinar para além das fronteiras do estado e do país.
} 
empoderamento na sua relação com o conhecimento, por meio de atuações concretas nas atividades escolares, deixando de lado o papel de "aluno universitário" para ser um "professor-mediador".

Dentre os subprojetos criados e em desenvolvimento e alguns, em processo de execução, destacam-se: Pluralidade Cultural - Máscaras, Oficina de Gravuras, Transferências e Impressões e o CINE Pibid - transversalizando saberes nas Artes Visuais. Outras inserções têm sido realizadas pelo nosso grupo, a pedido das coordenações dos Pibid da Dança (Grupo NUFOLK, Semana do Folclore) e Música (Oficina de sopapos, I Bienal das Artes e da Cidadania) da UFPel, dentre outros. Conforme apontamento realizado por Zely, "oferecer oficinas [poderá] melhorar nosso repertório para as escolas e dar mais ideias reais de como trabalhar em sala de aula" (AVALIAÇÃO, $1^{\circ}$ semestre 2015).

O primeiro subprojeto, que já tem sido desenvolvido desde 2014 e com maior efetividade em 2015, trata dos temas da Pluralidade Cultural e Meio Ambiente e foram geradores de importantes reflexões nos encontros do grupo. O objetivo do projeto é trabalhar as Artes Visuais como mediadora de contextos - Universidade e comunidade escolar propondo formas de desconstruir preconceitos enraizados no indivíduo, presentes na educação básica e em todas as outras esferas sociais, tratando da Arte Brasileira, em especial a AfroBrasileira e a Indígena (Figura 1).

O projeto desenvolve proximidade com as leis 10.639/2003 e 11.645/2008 que tornam obrigatório o estudo da história e cultura afro-brasileira e indígena nos estabelecimentos de ensinos fundamentais e médios. A exclusão da pessoa negra no processo educativo é evidenciada pela história da educação brasileira. A problemática da carência de abordagens históricas sobre as trajetórias educacionais dos negros no Brasil revela que não são os povos que não têm história, mas há os povos cujas fontes históricas, ao invés de serem conservadas, foram destruídas nos processos de dominação (CRUZ, 2005). Portanto, é imprescindível que esta temática seja trabalhada no contexto do Pibid, que busca unir o cotidiano dos alunos e escolas com a Universidade. 
RELACult - Revista Latino-Americana de Estudos em Cultura e Sociedade

Revista Latinoamericana de Estudios en Cultura y Sociedad | Latin American Journal of Studies in Culture and Society V. 02, nº 03, set-dez., 2016, p. 22-32 | periodicos.claec.org e-ISSN Atual: 2525-7870|e-ISSN Anterior: 2447-018X

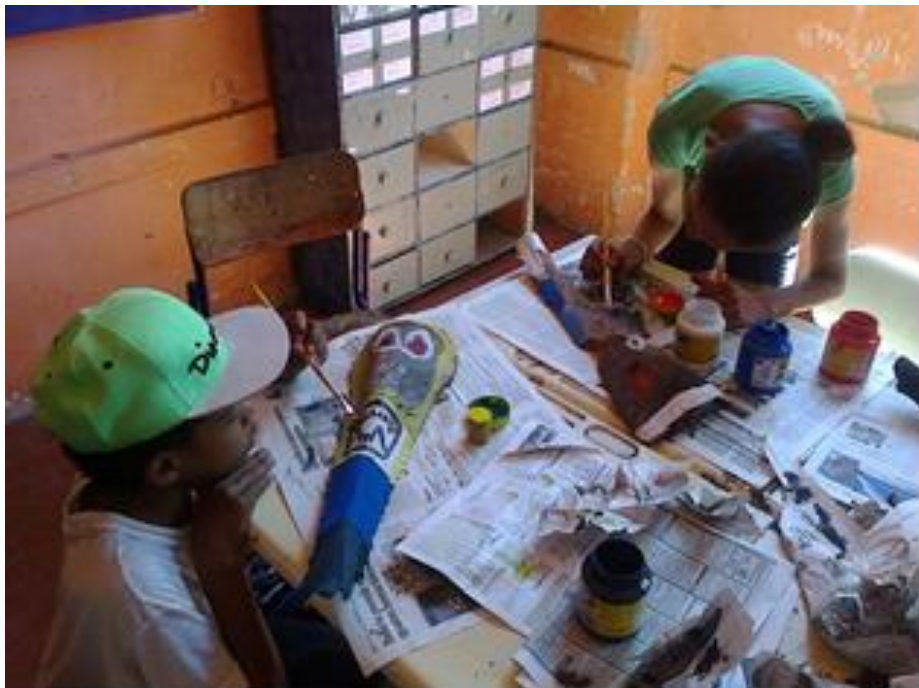

Figura 1: Na imagem à esquerda, 1: Alunos confeccionando máscaras com casacas de árvores - Semana da Consciência Negra, EEEF D. Joaquim F. de Mello, Pelotas, 2014. Fotografia: Thiago Plasa.

As oficinas desenvolvidas buscam, por meio da construção de máscaras com materiais alternativos, como cascas de árvores e materiais recicláveis, resgatar a cultura negra brasileira na formação da sociedade nacional, como também a importância do estudo dos povos indígenas brasileiros. Com esta atividade, buscamos também auxiliar na construção do senso crítico dos alunos em relação a sua identidade e formação cultural, rompendo com preconceitos existentes em nossa sociedade (Figura 2).

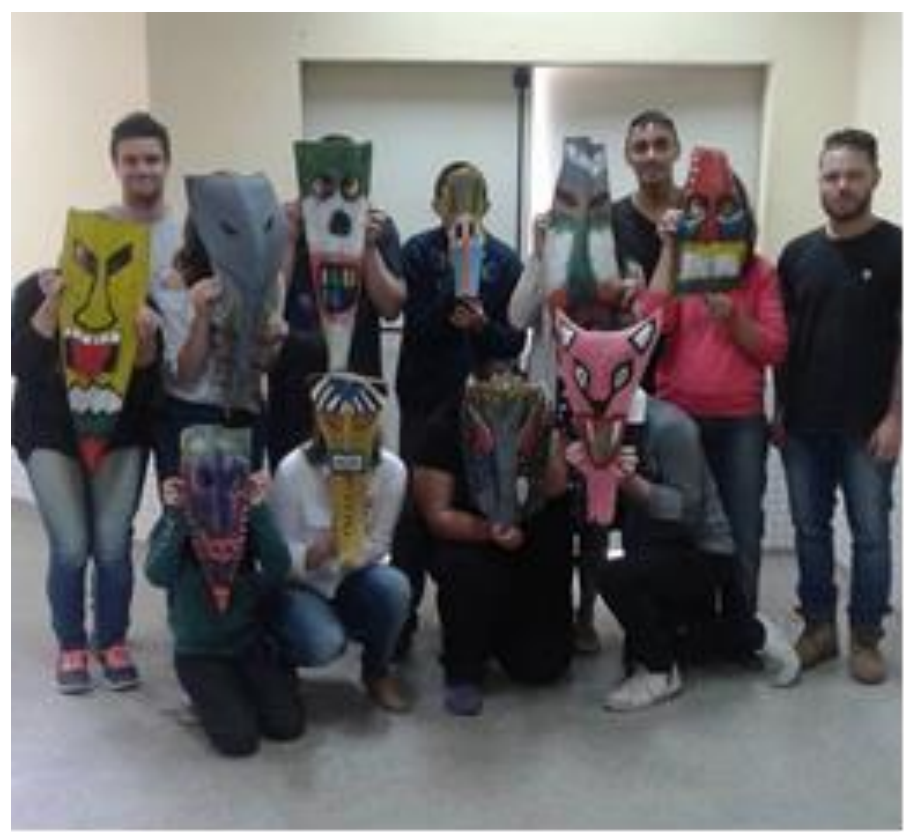


RELACult - Revista Latino-Americana de Estudos em Cultura e Sociedade e-ISSN Atual: 2525-7870 | e-ISSN Anterior: 2447-018X

Figura 2: Acadêmicos da Licenciatura em Matemática (UFPel), bolsistas e alunos do PIBID/Artes Visuais e Licenciatura em Artes Visuais, IV Ciclo de Oficinas de Matemática e outras Linguagens, out. 2015. Fotografia: Márcia da Fonseca.

Em relação ao subprojeto Pluralidade Cultural - Máscaras, o bolsista Ericsson avalia que "uma tarefa que ficou pendente foi o desenvolvimento de material teórico em cima dos resultados obtidos na oficina, no entanto nessas férias de julho tenho trabalhado nesse material para que possa ser levado ao ENEARTE (Encontro Nacional dos Estudantes de Artes) e a outros eventos acadêmicos que ocorrerão no decorrer desse segundo semestre letivo" (AVALIAÇÃO, $1^{\circ}$ semestre 2015). Percebemos, por esta e outras avaliações, como a de Lauro "[necessitamos] participar de eventos apresentando os subprojetos do Pibid (urgente!)”, que ainda temos uma trajetória a ser percorrida, no tocante à escrita e expressão de ideias em relação às práticas desenvolvidas no projeto (AVALIAÇÃO, $1^{\circ}$ semestre 2015).

O subprojeto Oficina de Gravuras, Transferências e Impressões iniciou em 2014, ainda no primeiro semestre e se encontra em andamento. Tem como objetivo a experimentação de transferências em diversos suportes, o conhecimento do histórico da Gravura e as técnicas básicas de gravação e experimentação de matrizes, como o piso vinílico, bases alternativas e a gravura verde, desenvolvendo impressões em suportes variados (Figuras 3 e 4).

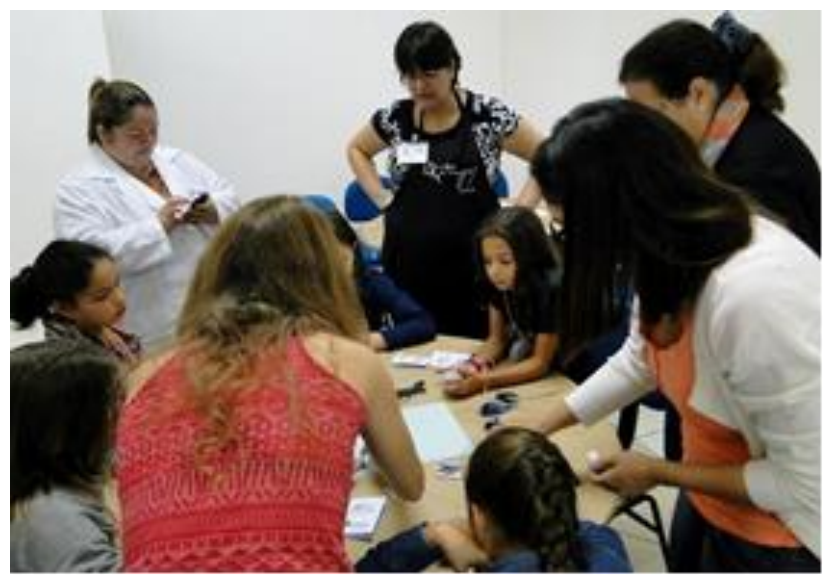

Figura 3: Oficina de Gravura na Semana dos Museus no MALG - Museu de Arte Leopoldo Gotuzzo (UFPel), maio 2015. Fotografia: Shayda Peres. 
RELACult - Revista Latino-Americana de Estudos em Cultura e Sociedade

Revista Latinoamericana de Estudios en Cultura y Sociedad | Latin American Journal of Studies in Culture and Society

V. 02, nº 03, set-dez., 2016, p. 22-32 | periodicos.claec.org e-ISSN Atual: 2525-7870|e-ISSN Anterior: 2447-018X

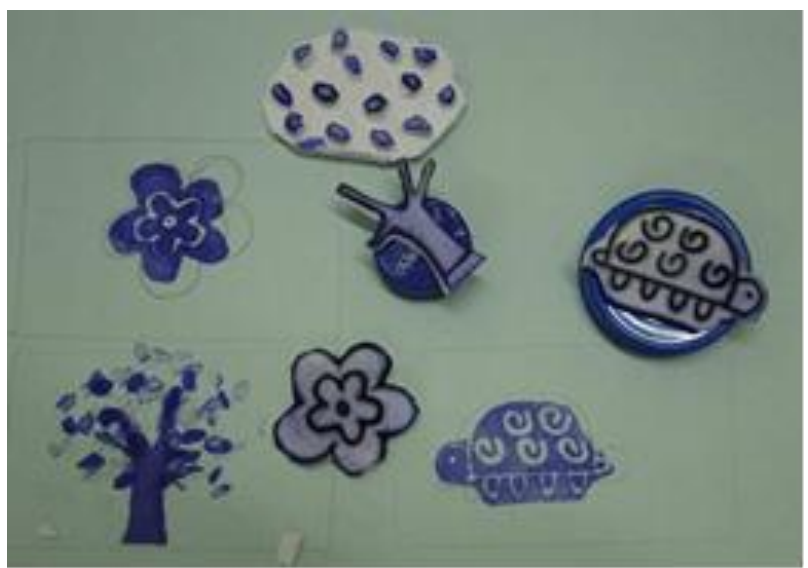

Figura 4: Matrizes e impressões realizadas com borrachas. Fotografia: Shayda Peres.

A Oficina de Gravuras, Transferências e Impressões trabalha com o Tema Meio Ambiente, visando reutilizar materiais descartáveis, como a caixa de leite tetra pak, papelões e outras materialidades alternativas. A oficina tem sido desenvolvida nas escolas, em museus e na universidade (Figura 5).

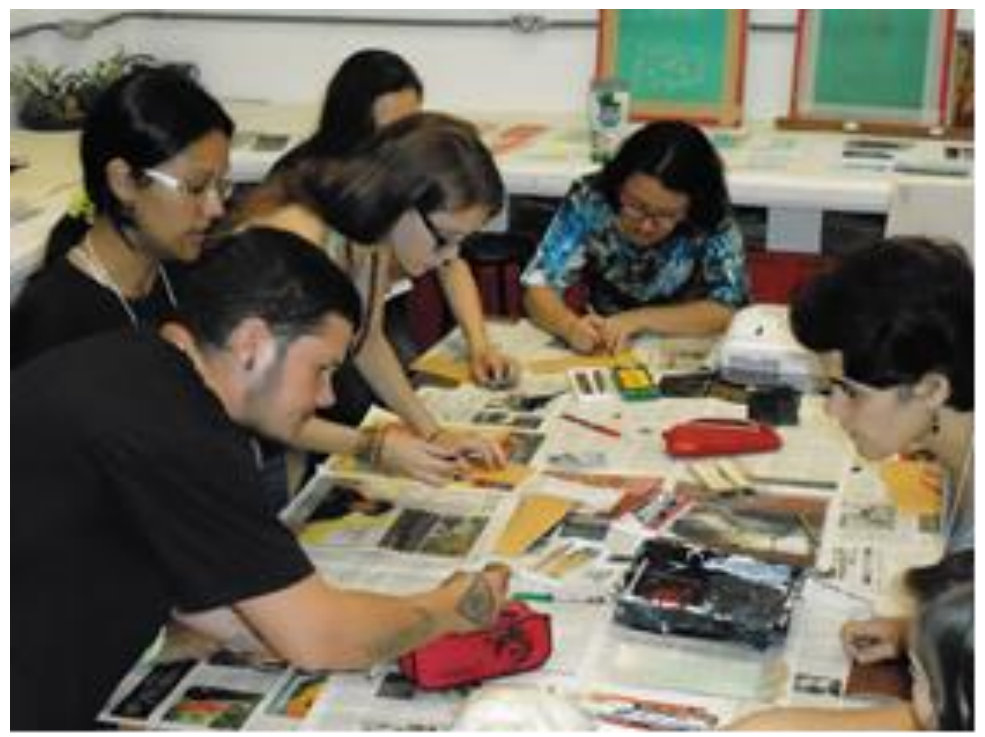

Figura 5: Bolsistas/Pibid e Professores no III Seminário do Pibid - UFPel, dez. 2014. Fotografia: Maristani Zamperetti.

Entendendo que a formação política dos acadêmicos deve ser considerada em sua formação inicial, o Pibid como política pública permitiu que as universidades revisassem sua visão diante das licenciaturas, entendidas, por vezes como um espaço desmerecido e esquecido, relacionados com a sensação de incompetência e a desistência da profissão docente 
(FERREIRA; REALI, 2005). Desta forma, a participação dos bolsistas nos movimentos de cunho político são relevantes para sua formação docente.

Podemos citar ainda a importância da Mobilização Nacional, em defesa do programa PIBID e da Formação de Professores (\#somostodospibid ${ }^{3}$ ), realizada no dia 15 de outubro. Entendendo que o Pibid é um espaço/tempo significativo no âmbito das políticas públicas em âmbito nacional, os bolsistas da UFPel estiveram reunidos no Largo do Mercado Público da nossa cidade (Figura 6).

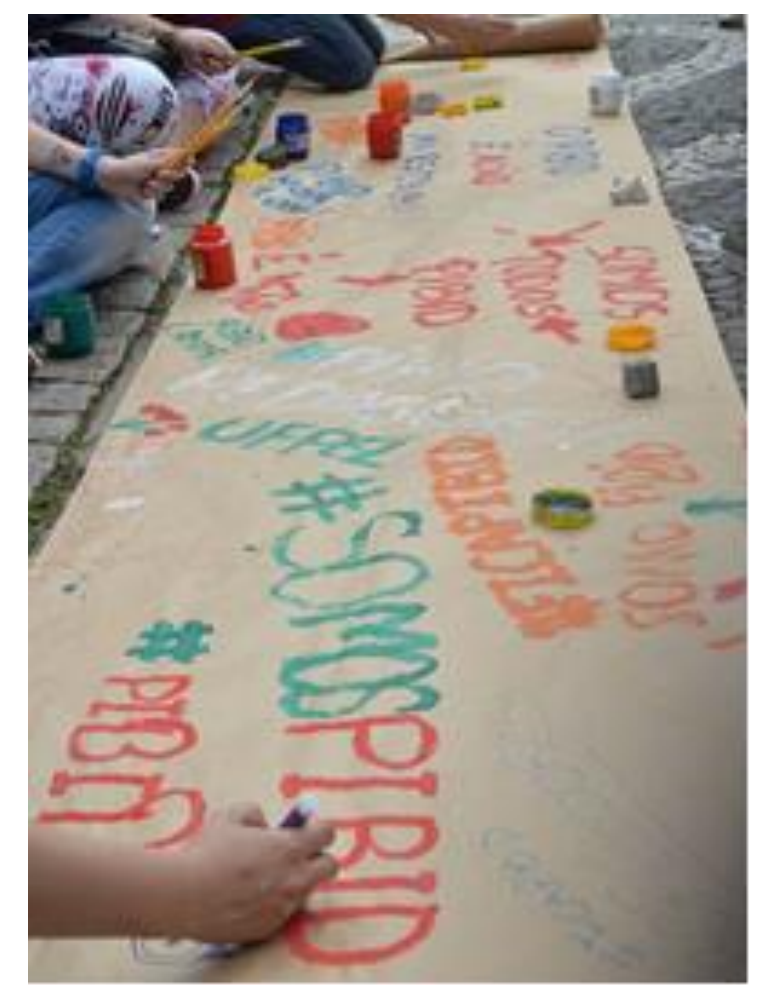

Figura 6: Faixa criada por licenciandos da UFPel para a Mobilização Nacional, em defesa do programa PIBID. Fotografia: Liz Dias, 2015.

\section{Breves conclusões}

Percebemos que o Projeto tem sido acolhido de forma positiva pelas escolas estaduais atendidas, alunos e comunidade escolar em geral, gerando grande receptividade em relação aos acadêmicos do Curso de Artes Visuais - Licenciatura. A arte e seu ensino podem colaborar na elaboração de novas ideias sobre os fatos do cotidiano, que não sejam encaradas

\footnotetext{
${ }^{3}$ Grupo do Facebook criado para a defesa do Pibid: https://www.facebook.com/Fica-PIBID-454188994748895/
} 
como verdades absolutas, mas sim, sendo vistas como mutáveis e provisórias, oxigenando o ambiente escolar, com alegria e criatividade. Afetividade, companheirismo, respeito e cooperação têm sido elencadas como características de crescimento no grupo, promovendo maior diálogo e tolerância à diversidade dentro e fora da escola.

Por meio da implementação de ações educacionais na escola, a prática investigativa tem sido estimulada no grupo e é possível observar uma aproximação maior dos bolsistas com a escrita acadêmica e a necessidade de registros de suas práticas. Verificamos um aumento no desenvolvimento e valorização de pesquisas na área da Arte/Educação por parte dos licenciandos pibidianos, em especial a partir do segundo semestre deste ano. Ocorreram participações no Congresso de Iniciação Científica da universidade como também em outros eventos, assim, pensamos que o Pibid contribuiu para o envolvimento dos alunos com pesquisas relacionadas ao ensino de suas áreas específicas. Assim, está ocorrendo uma maior valorização da pesquisa na formação de professores.

A valorização da pesquisa em sala de aula para o acadêmico promove a integração das diversas áreas do conhecimento em uma perspectiva interdisciplinar. Assim, a colaboração do Pibid - Artes Visuais para a parceria Escola-Universidade se mostra na conjugação dos diferentes contextos, proporcionando reverberações nos dois campos de atuação para os universitários e seus professores-coordenadores, como também para os professoressupervisores e seus alunos.

Verificamos grandes avanços na implementação dos subprojetos e do próprio projeto do Pibid na nossa área. A partir do relato publicado (ZAMPERETTI, 2014) foi possível perceber a ocorrência de um amadurecimento no grupo, destacando o fator da autonomia como potencial aflorado no mesmo. Talvez, pela característica do projeto institucional ser interdisciplinar, tenham ocorrido interações frutíferas com as mais diversas áreas de conhecimento das licenciaturas da universidade, promovendo crescimento intelectual e experiências estéticas, produzindo a ampliação de aprendizagens para o futuro docente no que tange às suas inserções na realidade escolar.

\section{Referências}

ASSMANN, Hugo. Reencantar a Educação. Rumo à sociedade aprendente. 8.ed. Petrópolis: Vozes, 2004.

BRASIL.SECRETARIA DA EDUCAÇÃO FUNDAMENTAL. Parâmetros Curriculares Nacionais: apresentação dos temas transversais, ética. Brasília: MEC/ SEF, 1997. 
CRUZ, Mariléria dos Santos et al. (orgs.). História da educação do Negro e outras histórias. Brasília: SECAD, 2005.

FERREIRA, L.; REALI, A. Aprendendo a ensinar e a ser professor: contribuições e desafios de um programa de Iniciação a Docência para Professores de Educação Física. Trabalho apresentado à 28. Reunião da ANPEd, Caxambu, 2005.

FREIRE, Paulo. Pedagogia da Autonomia. 30 ed. São Paulo: Paz e Terra, 2004.

GOYA, Edna de Jesus. O Pibid - A formação do professor de Artes Visuais e as atividades de ensino e pesquisa nas escolas municipais de Goiânia - segunda fase do Ensino Básico.

In: II ENCONTRO INTERINSTITUCIONAL DO PIBID \& III ENCONTRO INSTITUCIONAL PIBID-UFRGS. [Anais do...] Porto Alegre, 01-02 mar, 2011, 08p. Disponível em: http://www.ufrgs.br/prograd/pibid/anais-do-evento/salas-dedebate/O\%20PIBID\%20\%20A\%20formacao\%20do\%20professor\%20de\%20artes\%20visuais $\% 20 \mathrm{e} \% 20$ as $\% 20$ atividades $\% 20 \mathrm{de} \% 20 \mathrm{ensino} \% 20 \mathrm{e} \% 20$ pesquisa $\% 20$ nas $\% 20$ escolas $\% 20$ muni cipais\%20de\%20goiania\%20-\%20segunda\%20fase\%20do\%20ensino\%20basico.pdf Acesso em: 02 nov. 2015.

MACEDO, Elizabeth Fernandes de. Os Temas Transversais nos Parâmetros Curriculares Nacionais. Química Nova na Escola - Temas Transversais. Seção Espaço Aberto. N. 08, nov. 1998, p. 23-27. Disponível em: http://qnesc.sbq.org.br/online/qnesc08/espaco.pdf Acesso em: 01 nov. 2015.

NÓVOA, António (coord.). Os professores e a sua formação. 3.ed. Lisboa: Dom Quixote, 1997.

NÓVOA, António. Professores: Imagens do futuro presente. Lisboa: EDUCA, 2009.

PIMENTA, Selma Garrido. Pesquisa-ação crítico-colaborativa: construindo seu significado a partir de experiências com a formação docente. Educação e Pesquisa. São Paulo, v. 31, n. 3, p. 521-539, set./dez. 2005.

SOUZA, A. D. R. de; ZAMPERETTI, Maristani Polidori. Responsabilidades do artista educador nas reapropriações do consumo-descarte: experiências no PIBID Artes Visuais - UFPel. In: II SEMINÁRIO INTERNACIONAL ENSINO DA ARTE: culturas e práticas do cotidiano. [Anais do...] Pelotas, 14-16 out, 2015, p. 325-330.

ZAMPERETTI, Maristani Polidori. Formação docente no Pibid Artes Visuais - impressões iniciais de uma pesquisa. In: $24^{\circ}$ SEMINÁRIO NACIONAL DE ARTE E EDUCAÇÃO: os desafios do professor de arte no mundo contemporâneo. [Anais do...] Montenegro, v.24. 2014. p.222-228. 